\title{
Caractérisation des chevaux de concours hippique français d'après leur estimation génétique par un BLUP modèle animal
}

\author{
A Tavernier \\ Station de génétique quantitative et appliquée, Institut national de la recherche agronomique, \\ 78350 Jouy-en-Josas, France
}

(Reçu le 16 octobre 1989 ; accepté le 10 janvier 1990)

\begin{abstract}
Résumé - La valeur génétique des chevaux pour le concours hippique est maintenant estimée par un BLUP. Cette méthode permet de prendre en compte toutes les performances de tous les apparentés du cheval et de tenir compte en partie des différentes conditions de milieu : effet maternel, élevage, âge, sexe, année de la performance, conditions d'exploitation. La variable utilisée pour exprimer la performance est le logarithme du gain annuel. L'indice est exprimé en points d'écart type phénotypique $\left(\sigma_{\rho}=20\right)$. De cette évaluation se dégage la première description chiffrée de notre élevage en fonction de l'objectif de sélection représenté par la réussite en concours de saut d'obstacle. Dans cette orientation, le Selle-Français a 0,5 écart type génétique d'avance sur l'Anglo-Arabe et le département de la Manche a lui-même 0,5 écart type génétique d'avance sur l'ensemble des régimes d'élevage du Selle-Français. L'intensité de sélection demeure toutefois faible et le recours à des races extérieures sélectionnées sur la course apparaît de plus en plus aléatoire pour le développement de cette aptitude au saut. Chez les étalons, la sélection sur performance est bien trop laxiste (supériorité moyenne de 7 points) et reste peu précise (52\% n'ont qu'une année de performance). Chez les poulinières, elle devrait être plus généralisée (29\% seulement des juments sont testées). Le progrès génétique n'est, de ce fait, que de 1,9\% d'écart type phénotypique par an en Selle-Français (soit 0,37 point par an) et 0,7\% en Anglo-Arabe (soit 0,14 point par an). La structure démographique de ces populations autorise un progrès de 0,85 point par an chez les Selle-Français et de 0,47 point par an chez l'Anglo-Arabe ; ce qui laisse une importante plage de progrès possibles. Dans cet objectif, il serait souhaitable que le BLUP des étalons soit $\geq+19$ et celui des poulinières $\geq$ +4 indépendamment de leur coefficient de détermination. Toutefois, une procédure de sélection nuancée en fonction du coefficient de détermination est aussi proposée. II apparaît malgré tout, dans cette direction d'élevage, un handicap trop fort pour l'Anglo-Arabe pour lequel une redéfinition des objectifs semble nécessaire.
\end{abstract}

cheval d'obstacle / concours hippique / gain / BLUP / indice / sélection

Summary - Breeding characteristics of the French jumping horses using a BLUP animal model. The breeding value of French horses for jumping is now estimated by the BLUP method. This method takes into account all records on dam, sire and breeding background, and environmental influence such as maternal inheritance, herd effect, age, sex, year of recording and management conditions. The variable utilized is the logarithm of annual earnings. Breeding evaluation is expressed setting a value of 20 to one phenotypic standard deviation. Characteristics of the population are stressed. The Selle-Français horse has half a genetic standard deviation more than the AngloArab and horses from the department of the Manche (Normandy) also have half a genetic standard deviation more than other Selle-Français from other breeding areas. However, selection intensity is still weak, especially in males and the use of breeds specialized in racing is disadvantageous. In stallions, selection according to performance is not rigorous enough (average superiority of 7 points) 
and too imprecise (52\% of stallions have only one year of performance). Selection according to performance should become more widespread in mares (only 29\% of broodmares have records). The genetic trend is only $1.9 \%$ of phenotypic standard deviation in the Selle-Français and $0.7 \%$ in the AngloArab. To improve this situation, stallion BLUP rating should be $\geq+19$ and broodmare rating $\geq+4$. A selection table for stallions based on determination coefficients is also proposed. However, for the Anglo-Arab, a new definition of breeding objectives is required.

jumping horse / winning / BLUP / breeding evaluation / selection

\section{INTRODUCTION}

Les objectifs de production d'un cheval de selle peuvent être variés. Beaucoup de nos voisins européens visent un cheval polyvalent qu'ils sélectionnent au moyen du contrôle en station de différentes aptitudes (Philipsson et al, 1988 ; HedebroVelander et al, 1989 ; Huizinga et al, 1989 ; Bruns, 1989 ; Jensen et Staun, 1989). Cela ne va pas sans poser des problèmes de corrélations entre caractères (Bruns, 1987 ; Huizinga et Van Der Meij, 1988). Nous pensons qu'il est plus efficace de s'orienter vers un objectif précis assorti d'une mesure objective de la performance. L'estimation de la valeur génétique des chevaux à partir de leurs performances en concours hippique est réalisée en France depuis 1972. Nous disposons ainsi d'une mesure standardisée de la performance appelée indice annuel, et nous calculons aussi un indice sur descendance pour les étalons (Langlois, 1980). Depuis 1985 (Tavernier, 1985), la méthode d'estimation est basée sur un BLUP (Best Linear Unbiased Predictor) en modèle individuel qui permet de donner un indice à tous les chevaux, calculé à partir de toute leur parenté. Les étalons, les juments, les poulains, les jeunes performants sont alors estimés sur une même base. Depuis, l'Allemagne et la Hollande se sont attachées à mettre en œuvre le même type d'indexation sur leur propres chevaux (Huizinga et Van Der Meij, 1988; Meinardus et Bruns, 1987). Notre propos est ici de caractériser l'élevage français poûr. l'aptitude au concours hippique grâce au BLUP et d'en déduire les possibilités de progrès dans cette orientation.

\section{MATÉRIEL ET MÉTHODE}

La technique de calcul utilisée a été décrite pour les chevaux de sport (Tavernier, 1988). Nous n'en ferons donc qu'un bref rappel. Le choix du critère de mesure de la performance et des facteurs de variation a été discuté par Langlois (1980).

\section{Modèle}

La performance en concours hippique est mesurée par le logarithme du gain annuel du cheval (Langlois, 1980). Ce logarithme permet de rétablir l'échelle distendue des gains, puisque ceuxci sont distribués de façon exponentielle par rapport au niveau de la performance : un cheval gagne environ $79 \%$ de ce que gagne le cheval placé avant lui. Le gain est la meilleure mesure de la réussite du cheval en concours hippique. Cette performance est expliquée par le modèle linéaire :

$$
y=X b+Z u+W m+Z p+e
$$

avec :

$-y:$ vecteur des observations (logarithme du gain annuel) ;

$-b$ : vecteur des effets fixes (sexe et classe d'âge-année) :

- u : vecteur des valeurs génétiques additives individuelles ;

- $m$ : vecteur des effets "maternel-élevage" (effet de milieu commun aux descendants d'une même mère) ; 
- $p$ : vecteur des effets du milieu commun aux différentes performances d'un même cheval ;

- e : vecteur de l'erreur résiduelle ;

$-X, Z, W$ : matrices d'incidence.

Les effets connus du milieu (effets fixes) sont le sexe - 2 niveaux : femelle et mâle (ou hongre) - et une combination de l'âge et de l'année. II y a 5 classes d'âge : 4 ans, 5 ans, 6-7 ans, 8-9-10 ans, 11 ans et plus, croisées avec 17 années de performances. La combinaison de l'âge et de l'année est nécessaire car la politique de dotation des épreuves en fonction de l'âge varie dans le temps et se combine aux effets de l'inflation. L'effet "maternel-élevage" est une interprétation de la différence trouvée entre les composantes paternelle et maternelle de la variance. Cette différence est due à un effet de milieu commun aux différents descendants d'une même mère. Soixante-dix-huit pour cent des élevages de chevaux de selle ne comprennent qu'une seule jument, l'effet maternel strict se confond donc avec l'influence de l'éleveur : alimentation, soins, mise en valeur des produits. L'effet de milieu commun aux différentes performances d'un même cheval représente la différence entre la corrélation entre 2 performances (répétabilité) et l'héritabilité ajoutée à l'effet maternel. II est expliqué par le milieu constant après l'élevage, dont bénéficie le cheval durant plusieurs années de suite.

Les espérances des effets de ce modèle linéaire sont :

$$
E\left[\begin{array}{c}
y \\
u \\
m \\
p \\
e
\end{array}\right]=\left[\begin{array}{c}
x b \\
0 \\
0 \\
0 \\
0
\end{array}\right]
$$

La matrice de variance-covariance est :

$$
V\left[\begin{array}{c}
u \\
m \\
p \\
e
\end{array}\right]=\left[\begin{array}{cccc}
A \sigma_{u}^{2} & 0 & 0 & 0 \\
0 & 1 \sigma_{m}^{2} & 0 & 0 \\
0 & 0 & 1 \sigma_{p}^{2} & 0 \\
0 & 0 & 0 & 1 \sigma_{\mathrm{e}}^{2}
\end{array}\right]
$$

avec

$\sigma_{u}^{2}=h^{2} \sigma_{y}^{2} ; \sigma_{m}^{2}=v \sigma_{y}^{2} ; \sigma_{p}^{2}=\left(r-v-h^{2}\right) \sigma_{y}^{2} ;$

$\sigma_{\theta}^{2}=(1-r) \sigma_{y}^{2}$

A : matrice de parenté; 1 : matrice identité;

$h^{2}$ : héritabilité ; $r$ : répétabilité ; $v$ : fraction de la variance phénotypique due à la composante maternelle-élevage.
La matrice de parenté comprend l'ensemble des animaux, reproducteurs ou non, performants ou non. Les lignes de la matrice $Z$ correspondant aux animaux non performants ne contiennent que des 0 .

\section{Équations et résolution du modèle mixte}

Les équations du modèle mixte sont :

$\left[\begin{array}{cccc}X^{\prime} X & X^{\prime} Z & X^{\prime} W & X^{\prime} Z \\ Z^{\prime} X & Z^{\prime} Z+t_{1} A^{-1} & Z^{\prime} W & Z^{\prime} Z \\ w^{\prime} X & W^{\prime} Z & W^{\prime} W^{\prime} t_{2} l & W^{\prime} Z \\ Z^{\prime} X & Z^{\prime} Z & Z^{\prime} W & \left.Z^{\prime} Z+t_{3}\right)\end{array}\right]\left[\begin{array}{c}b \\ u \\ m \\ p\end{array}\right]=\left[\begin{array}{c}X^{\prime} y \\ Z^{\prime} y \\ W^{\prime} y \\ Z^{\prime} y\end{array}\right]$

avec

$t_{1}=\frac{(1-r)}{h^{2}} ; t_{2}=\frac{(1-r)}{v} ; t_{3}=\frac{(1-r)}{\left(r-h^{2}-v\right)}$

Les effets $p$ et $m$ sont absorbés facilement grâce à la structure diagonale des matrices $\left(W^{\prime} W+t_{2} l\right)$ et $\left(Z Z+t_{3} l\right)$. Le résultat de leur $a b-$ sorption se calcule directement à partir du nombre d'années de performances, des performances de chacun et de leurs sommes par individu et par mère. Les animaux non reproducteurs et les mères sont ensuite absorbés grâce à la structure particulière de l'inverse de la matrice de parenté. En regroupant les poulinières issues d'une même jument fondatrice (sans mère), on obtient une matrice "poulinière $x$ poulinière" bloc diagonale dont l'inversion est aisée. De même on regroupe les individus non reproducteurs par mère (Tavernier, 1988). La matrice finale ne comporte plus que les effets fixes et les étalonis. Elle est résolue par itération. Les solutions des poulinières et des non-reproducteurs absorbés sont obtenues par back-solving des équations à l'aide des solutions étalons et effets fixes.

La précision de ces estimations est obtenue par approximation (Van Raden et Freeman, 1985) de la décomposition par bloc de l'inverse de la matrice finale. Si on note linverse de la matrice finale :

$$
\left[\begin{array}{lll}
H & B^{\prime} & -1 \\
B & M
\end{array}\right]=\left[\begin{array}{ll}
T & S^{\prime} \\
S & F
\end{array}\right]
$$


avec $\mathrm{H}=$ bloc correspondant à la proche parenté de l'étalon dont on veut calculer la précision de l'estimation, on a :

$$
\mathrm{T}=\mathrm{H}^{-1}+\mathrm{H}^{-1} \mathrm{~B}^{\prime} \mathrm{FB} \mathrm{H} \mathrm{H}^{-1} \text {. }
$$

Dans le sens où $C \geq D$ veut dire que $(C-D)$ est positive semi-définie, on peut montrer que :

- une borne supérieure de $T$ est :

$$
\mathrm{H}^{-1}+\mathrm{H}^{-1} \mathrm{~B}^{\prime} \text { A B H-1; }
$$

- une borne inférieure de T est : $\mathrm{H}^{-1}$.

La borne supérieure de $T$ est approchée en utilisant la structure connue de parenté du bloc $\mathrm{H}$ et la parenté "moyenne" contenue dans $\mathrm{A}$. Les précisions des estimations des poulinières et des non-reproducteurs sont obtenues simplement par décomposition par bloc de l'inverse et utilisation des précisions des étalons.

\section{Matériel}

Les gains en compétitions équestres sont recueillis et informatisés par la Société des Steeple-Chases de France depuis 1972. La généalogie est gérée par le SIRE : Système d'identification répertoriant les Équidés. Les ancêtres de tous les performants sont recensés jusqu'aux naissances de 1945. Le fichier final comprend 5204 étalons, 46643 poulinières et 60235 non-reproducteurs. Après calcul des indices sur ce fichier informatif, nous avons estimé, par la moyenne des estimations de leurs parents, le reste des chevaux inscrits au SIRE, c'est-à-dire tous les non-performants et nonparents de performants nés depuis 1974 ainsi que leurs ancêtres. Le fichier complet comprend 565251 chevaux.

Les paramètres génétiques avaient été estimés par Langlois (1980) et Tavernier (1986) par analyse de variance du logarithme du gain annuel précorrigé pour les effets fixes estimés par un moindre carré. La répétabilité est de 0,45 , la composante maternelle-élevage est de 0,05 et l'héritabilité de 0,20.

\section{RÉSULTATS}

Tous les résultats sont exprimés en points de performance. La performance est standardisée avec un écart type de 20. La précision de l'indice est présentée sous la forme d'un coefficient de détermination qui varie de 0 à 1 .

\section{Estimation des effets fixes}

L'estimation des effets fixes âge-année obtenus par la résolution du modèle mixte s'appelle BLUE (Best Linear Unbiased Estimate). La fig 1 montre des divergences

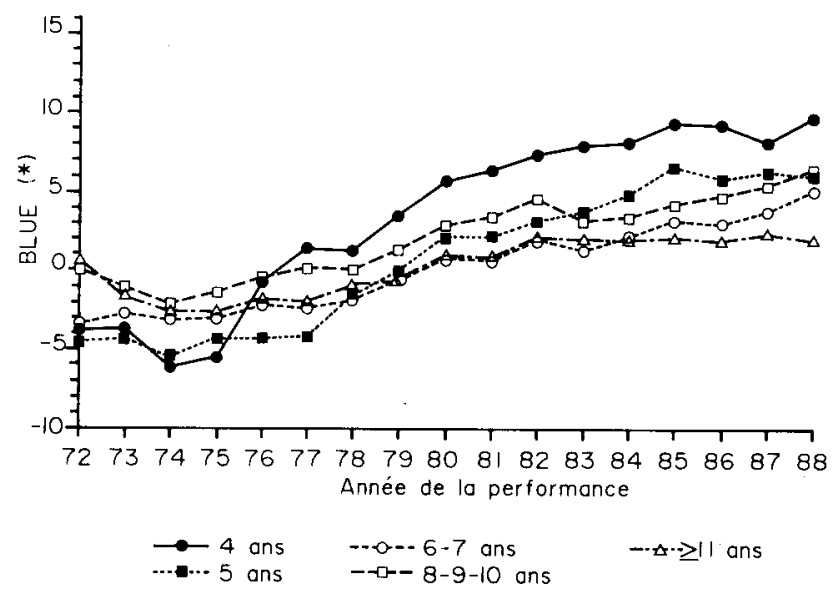

Fig 1. Estimation des effets fixes. ( $\left.{ }^{\star}\right)$ Le "0" est le niveau (âge 8-9-10 ans)-(année 1972). Standardisé par rapport à un écart type phénotypique de 20 points. 
dans l'estimation de l'effet de l'âge en fonction de l'année. En effet, les dotations varient non seulement en fonction de l'inflation (qui explique l'augmentation des estimations avec l'année) mais aussi en fonction de la politique sportive. Avant 1976, il n'y avait pas de circuit spécialisé avec des dotations particulières pour les jeunes chevaux. Cela explique que les 4 ans, qui ont moins de «métier", gagnaient alors moins d'argent que leurs concurrents plus âgés. Maintenant, les épreuves de 4 ans sont protégées et largement dotées, ce qui explique l'effet très favorable de cette classe d'âge. Simultanément, bien que moins généralisé, s'est développé le circuit des chevaux de 5 ans. Dans le circuit "normal», les chevaux qui bénéficient à la fois de leur expérience et de leur forme sont les 8-9-10 ans. Les chevaux de 6-7 ans sont encore inexpérimentés et les chevaux de 11 ans et plus voient leur forme diminuer. Une tendance récente donne maintenant un avantage aux 6-7 ans par rapport aux 11 ans et plus.

L'estimation BLUE peut être comparée à l'estimation actuellement utilisée pour corriger les données (fig 2). Cette dernière estimation était un moindre carré réalisé chaque année sur un modèle ne comportant que des effets fixes. Les performances étaient standardisées intra-année. Les estimations des moindres carrés sont supérieures à celles du BLUE et ces écarts augmentent en fonction de l'année. Cette différence est due au progrès génétique. En effet, les différences entre niveaux d'effets fixes d'ordre génétique sont incluses dans l'estimation des moindres carrés, alors qu'elles doivent s'en séparer dans l'estimation par un BLUE. De la même manière, cet écart est plus important à 4 et, dans une moindre mesure, à 5 ans : les chevaux qui sortent en compétition à cet âge précoce et qui bénéficient des épreuves spéciales "jeunes chevaux" sont en fait un échantillon sélectionné de la population. De même, les chevaux qui, en revanche, sortent après 11 ans, sont un échantillon sélectionné : à cet âge, seuls les meilleurs restent. Ces nouvelles estimations sont donc plus justes que celles utilisées auparavant.

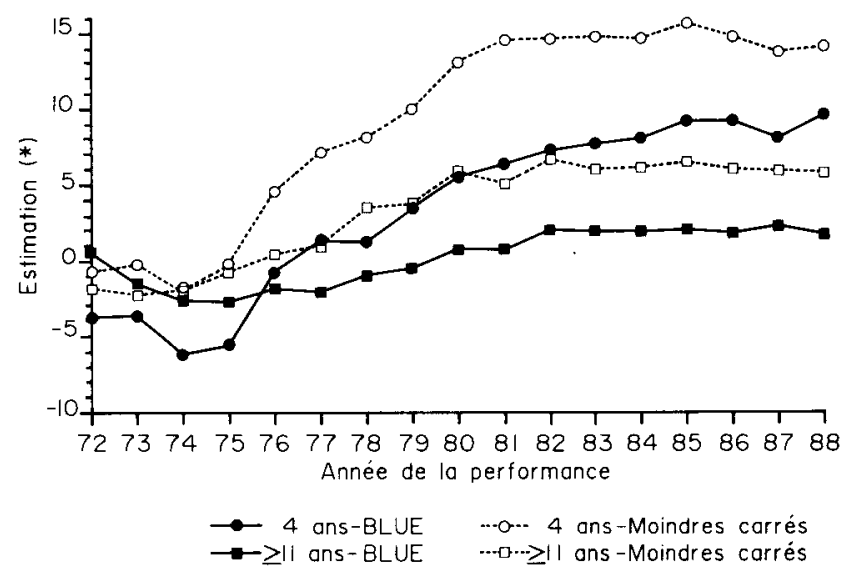

Fig 2. Comparaison des estimations des effets fixes : BLUE et moindres carrés. (") Le «0" est la classe (âge 8-9-10 ans)-(année 1972). Standardisé par rapport à un écart type phénotypique de 20 points. 


\section{Structure de la population}

Le " 0 " de référence correspond au niveau de la population de base : les chevaux de notre fichier dont on n'a pas remonté les parents.

Il existe en France 2 races de chevaux de selle qui possèdent un Stud-Book et peuvent prétendre à la production d'un cheval de concours hippique : le SelleFrançais (SF) et l'Anglo-Arabe (AA). En pratique, toutes les races enregistrées au SIRE peuvent participer aux compétitions, donc aussi bien les races spécialisées dans la course - Pur Sang (PS) et Trotteurs Français (TF) - que les chevaux issus de poulinières qui n'appartiennent pas à un Stud-Book et appelés "chevaux de selle" (CS). Ces 2 catégories représentent respectivement $15 \%$ et $12 \%$ des gagnants en concours hippique mais les populations globales sont estimées avec trop peu de précision pour donner des statistiques intéressantes.

La distribution des indices BLUP des naissances 1987 des chevaux SelleFrançais et Anglo-Arabes ( 8472 poulains) a pour moyenne 4,6 et pour écart type 6,2. Le quart de la population a un indice $\geq$ a $+9,10 \%$ à +14 , et $5 \%$ à +16 .
Chaque race est néanmoins bien individualisée. La distribution des indices BLUP des naissances 1987 de Selle-Français a pour moyenne $+5,3$ et pour écart type 6,3 pour 7063 poulains. Le maximum est de + 27,7 et le minimum de $-10,3$. Chez les Anglo-Arabes, la moyenne est de $+0,7$, l'écart type est de 4,0 pour 1409 poulains. Le maximum est de $+13,4$ et le minimum de $-9,2$ (fig 3). Ces statistiques illustrent les différences de vocation des 2 races. La première, le Selle-Français, se spécialise dans la production d'un bon cheval de concours hippique. La deuxième garde un objectif multiple (en plus du concours hippique : les courses, le concours complet) qui entraîne à la fois une moins bonne connaissance de la population (écart type faible) et un retard de déjà 4,5 points sur sa rivale, ce qui représente 0,5 écart type génétique.

Ces différences entres races recouvrent aussi des différences entre régions (fig 4). Dans la production d'Anglo-Arabes se situe principalement dans le Sud-Ouest dont la majorité des départements de cette région la moyenne des BLUP des chevaux nés en 1987 est inférieure à la moyenne nationale. Le Selle-Français est un rassemblement d'origines différentes. Ainsi, la Manche (moyenne + 9,7, 1279 nais-

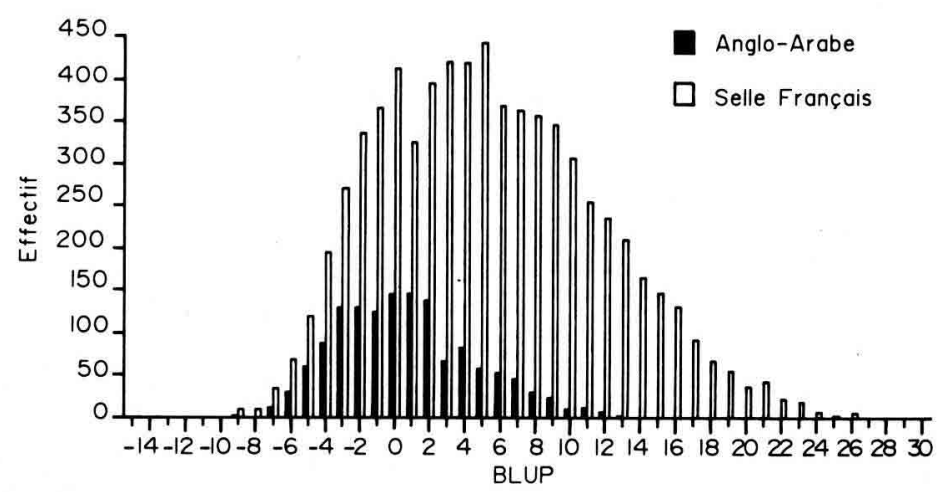

Fig 3. Distribution des indices BLUP des naissances de 1987. 


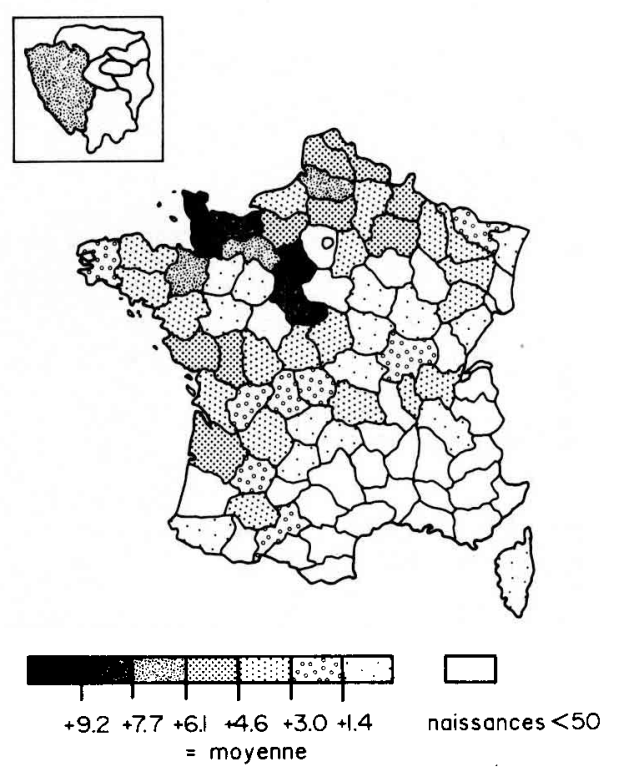

Fig 4. BLUP des naissances Selle-Français et Anglo-Arabes de 1987.

sances), le Calvados (moyenne $+8,3,392$ naissances) et l'Orne (moyenne $+7,3,261$ naissances) représentent la zone traditionnelle d'élevage du Selle-Français issu de l'Anglo-Normand. C'est lui qui a connu le plus bel essor dans la compétition équestre. La Manche a ainsi un avantage de 0,5 écart type génétique sur la moyenne de la population française. Après la Normandie et bien que beaucoup plus modestes en effectif, on trouve d'autres régions où le Selle-Français a une autre origine : le Nord représenté par la Somme, la Bretagne représentée par l'llle-et-Vilaine. Enfin, on voit poindre de nouvelles régions qui profitent de la proximité d'aggiomérations où se sont développés les sports équestres : les Yvelines, le Loir-et-Cher et l'Eure-et-Loir.

\section{Progrès génétique}

Le progrès génétique est mesuré par l'évolution de l'indice BLUP en fonction de l'année de naissance. Nous avons étudié cette évolution depuis les naissances de 1974 (date du début de l'enregistrement systématique de toutes les naissances) jusqu'à celles de 1987, incorporant toutes les performances depuis 1972 jusqu'à 1988 inclus.

Chez les Selle-Français, l'évolution est de 0,37 point par an. Chez les AngloArabes, elle est de 0,14 point par an donc 2,5 fois moindre. Les Chevaux de Selle (CS), partis de plus bas, rejoignent aujourd'hui les Anglo-Arabes en bénéficiant par les mâles (inscrits à un Stud-Book) du progrès réalisé dans les autres races. Leur évolution est de 0,18 point par an (fig 5). Grâce à ces estimations, on peut mesurer en temps l'écart qui sépare la population Selle-Français de la population AngloArabe. Les 4,6 points d'écart représentent 13 ans d'évolution pour le Selle-Français ou bien 33 ans pour l'Anglo-Arabe. Cette évolution est différente dans les diverses régions d'élevage françaises. On retiendra l'évolution du département de tête, la Manche, qui a connu un progrès beaucoup plus rapide dans les années $76-80$ puisque sa supériorité par rapport à l'ensemble de la population est passée de 3 à 5 points mais cet écart est maintenant stable, voire en légère diminution ( 4,4 points en 1987).

Ce progrès est presque exclusivement apporté par les mâles, les juments n'étant pas sélectionnées (fig 6 et 7). Les étalons Selle-Français sont sélectionnés de façon de plus en plus intensive avec le temps. Leur supériorité génétique est mesurée par la moyenne de l'écart à la population de chaque étalon pondéré par leur nombre 


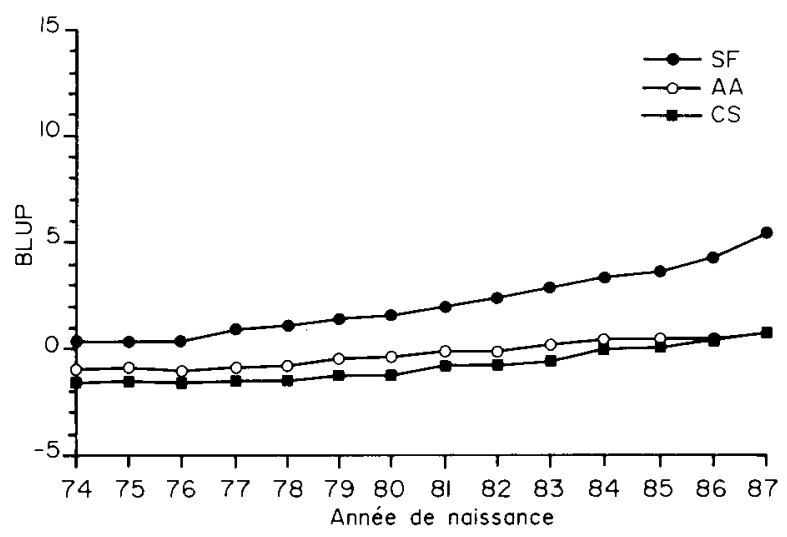

Fig 5. Évolution génétique des Selle-Français (SF), des Anglo-Arabes (AA) et des Chevaux de Selle (CS).

de descendants, ce qui représente leur véritable influence génétique. Leur supériorité moyenne est donc de 10,2 points. La sélection des étalons Anglo-Arabes évolue elle aussi favorablement, puisque la valeur génétique des étalons de cette race s'accroît et qu'ils présentent ainsi une supériorité moyenne par rapport à la population de 3,4 points (en dehors de l'année 1976 qui a été catastrophique). Les femelles présentent un avantage d'environ 0,2 point chez les Selle-Français et quasiment nul chez les Anglo-Arabes.

Historiquement, le Selle-Français est issu du croisement d'une jument assez épaisse, pour les travaux des champs ou la traction légère, avec des étalons de PurSang. Dans un premier temps, les qualités sportives sélectionnées sur la course du cheval de Pur-Sang ont apporté un plus au

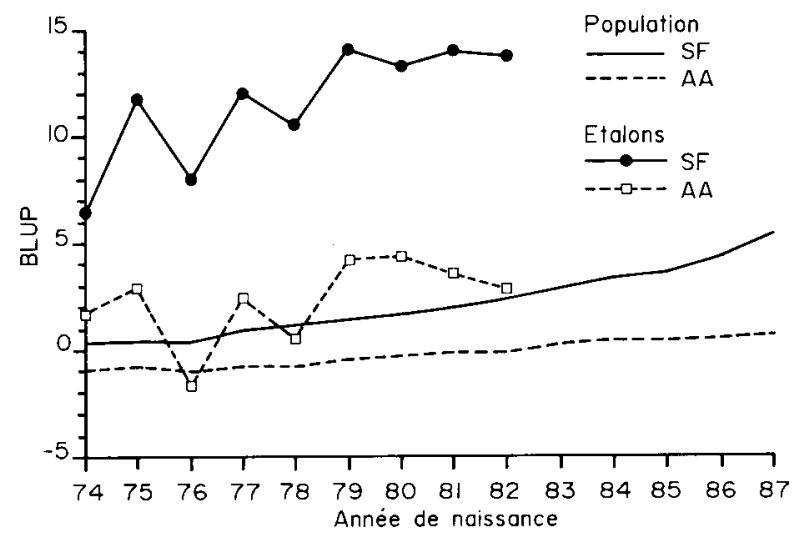

Fig 6. Évolution génétique des étalons. 


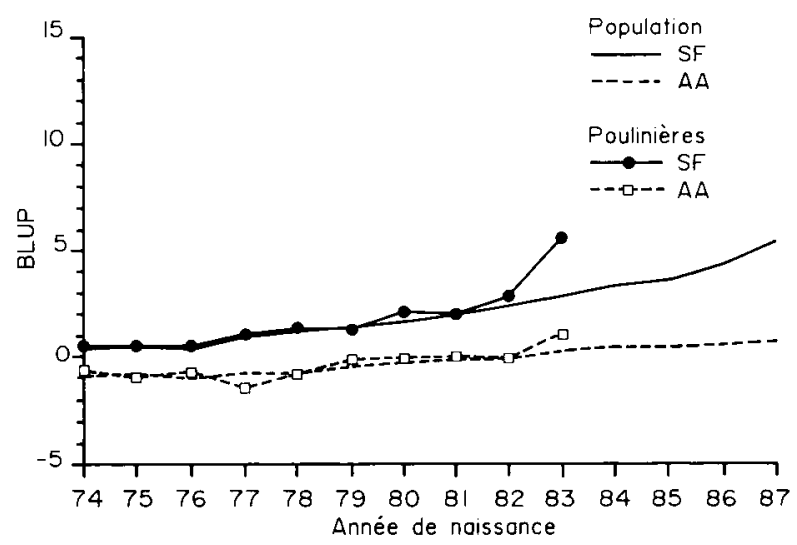

Fig 7. Évolution génétique des poulinières.

cheval de concours hippique qui commençait à se développer. L'influence que l'on observe maintenant du Pur-Sang (et de l'autre cheval de course : le Trotteur Français) est différente (tableaux I et II). Le Pur-Sang sélectionné sur la course a suivi une orientation divergente de la race maintenant plus déterminée du Selle-Français, même si ce dernier peut toujours être issu de croisements avec des Pur-Sang, des Trotteurs Français ou des Anglo-Arabes. L'influence "moyenne" de la race PurSang n'est plus favorable au concours hippique. Cette influence est mesurée par la moyenne de leur écart à la population dans laquelle ils reproduisent (SelleFrançais ou Anglo-Arabe) pondéré par leur nombre de descendants dans chacune de ces races (fig 8). D'autre part, les moyennes des Pur-Sang et des AngloArabes utilisés pour produire du SelleFrançais sont supérieures à celles de ceux de même race utilisés pour produire de l'Anglo-Arabe. Cela vient sans doute du fait que les Anglo-Arabes gardant un objectif de cheval de course, on a recours aux étalons de Pur-Sang et Anglo-Arabes sélectionnés pour cette aptitude. En revanche, les étalons de ce type ne sont plus employés en Selle-Français dès qu'ils sont reconnus comme non intéressants pour le concours hippique. En effet, le nombre de descendants produits par un étalon est fonction, en partie, de sa qualité.

Tableau I. Supériorité moyenne des reproducteurs Selle-Français (SF), Anglo-Arabes (AA), PurSang (PS) et Trotteurs (TF) parents de Selle-Français.

\begin{tabular}{lcccc}
\hline & $S F$ & $A A$ & $P S$ & $T F$ \\
\hline Étalons & $+10,2$ & $+1,5$ & $-2,0$ & $-1,4$ \\
Poulinières & $+0,2$ & $-1,2$ & $-3,6$ & $-3,2$ \\
\hline
\end{tabular}


Tableau II. Supériorité moyenne des reproducteurs Anglo-Arabes (AA) et Pur-Sang (PS) parents d'Anglo-Arabes.

\begin{tabular}{lll}
\hline & $A A$ & $P S$ \\
\hline Étalons & $+3,4$ & $-1,5$ \\
Poulinières & $+0,1$ & $-2,1$ \\
\hline
\end{tabular}

Les meilleurs étalons font davantage de descendants, comme en témoigne l'écart entre la moyenne brute des indices BLUP des étalons et cette moyenne pondérée par le nombre de descendants. Cet écart est de 1,1 point (SF) et 0,2 point (AA) en faveur de la moyenne qui tient compte du nombre de descendants, quel que soit l'âge de l'étalon. Chez les étalons de PurSang qui produisent en Selle-Français, l'écart n'est sensible que pour les étalons plus âgés ( 2 à 3 points pour ceux qui sont nés de 1974 à 1986 contre 0 point pour ceux nés de 1979 à 1981). L'utilisation du Pur-Sang en Selle-Français est donc bien raisonnée par les éleveurs mais ne peut l'être qu'après l'apparition des premiers produits alors que celle des étalons Selle-
Français peut l'être bien avant grâce à la parenté et aux performances.

\section{Sélection sur les performances}

La sélection du cheval de sport est une activité récente qui transforme le cheval d'usage ou le cheval d'arme en cheval de compétition. On a longtemps pensé que, malgré cette orientation nouvelle, il suffisait de sélectionner ces chevaux sur leur conformation. Depuis peu a germé l'idée d'une sélection sur performance, d'autant plus permise aujourd'hui qu'une indexation généralisée de la population est disponible. Quelle est la situation actuelle (saillies de 1988) en matière de contrôle des performances et quel est l'avantage moyen attaché aux chevaux testés en compétition ?

Chez les Selle-Français, $63 \%$ des étalons sont sortis en compétition, chez les Anglo-Arabes, $43 \%$. Le nombre moyen d'années de performance est de 2,6 et de 2,1 respectivement avec une forte proportion de chevaux qui ne sortent qu'une année : $52 \%$ en Selle-Français et $65 \%$ en Anglo-Arabes. Le niveau génétique des

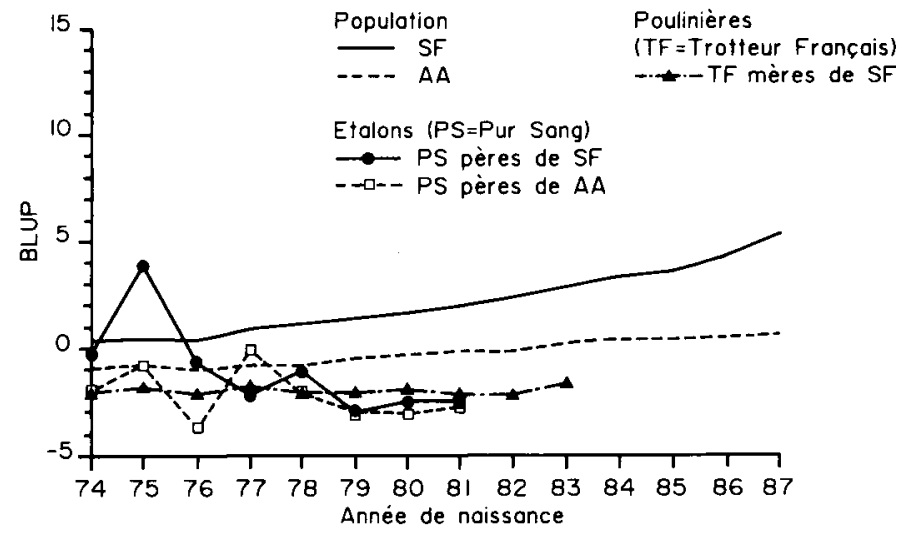

Flg 8. Évolution génétique des reproducteurs de races de course parents de chevaux de selle. 


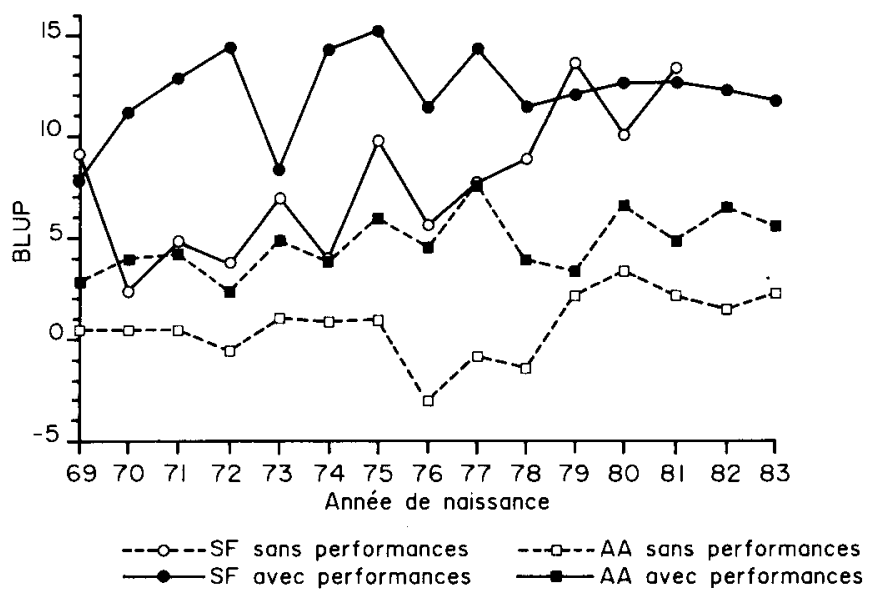

Fig 9. Indice BLUP des étalons Selle-Français et Anglo-Arabes en service en 1988.

étalons Selle-Français performants est en moyenne supérieur de 7 points à celui de leurs contemporains non performants, à l'exception de ces dernières années où les rares non-performants ne sont qu'à 2 points d'écart (fig 9). Chez les AngloArabes, les performants ont en moyenne un avantage de 4 points. La population des étalons Selle-Français rajeunit avec l'introduction de jeunes étalons tous performants (fig 10). Ainsi l'âge moyen au 1 er janvier 1988 des étalons performants est-il de 8,9 ans alors que celui des autres étalons est de 14,1 ans. Ce rajeunissement peut être temporaire : il provient actuellement de l'essor de l'étalonnage privé qui propose de jeunes performants ; la situation peut ensuite se stabiliser si l'ensemble de ces étalons continue à saillir. Inversement, le prestige de ces jeunes étalons et donc leur utilisation - pourra fortement diminuer quand de nouveaux jeunes arri-

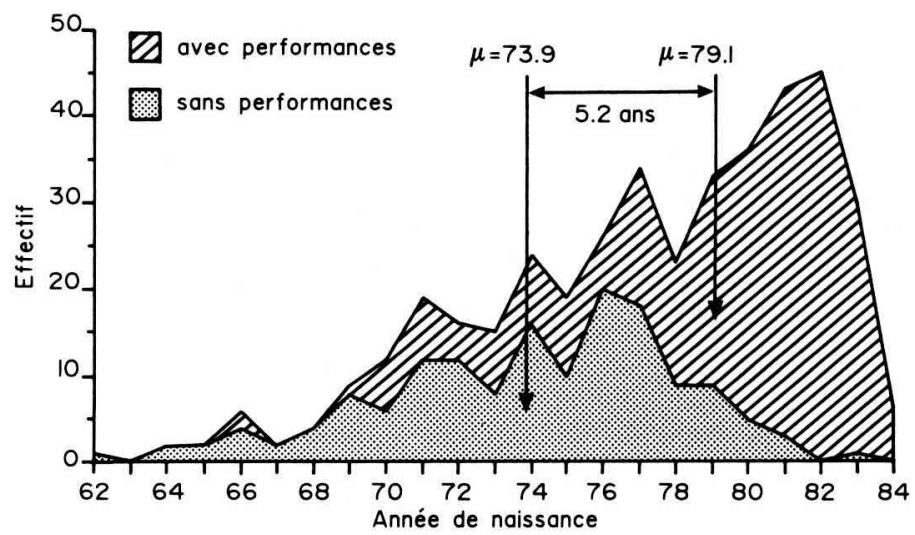

Fig 10. Année de naissance des étalons Selle-Français en activité en 1988. 


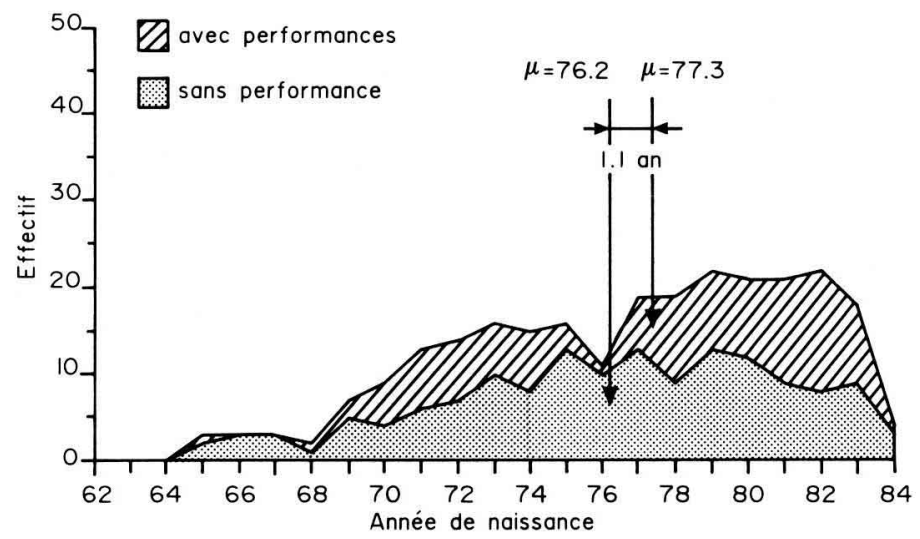

Fig 11. Année de naissance des étalons Anglo-Arabes en activité en 1988.

veront ; la population, dans ce cas, restera jeune. En Anglo-Arabes, l'écart est beaucoup moins sensible $(10,7$ ans et 11,8 ans respectivement) et il n'y a pas la même augmentation du nombre d'étalons (fig 11).

La proportion de juments sorties en compétition est de $29 \%$ chez les SelleFrançais et $22 \%$ chez les Anglo-Arabes. La valorisation des juments - laissée à l'initiative d'éleveurs - est donc assez similaire dans les deux races. La moyenne du nombre d'années de performance est de 3,1 pour les Selle-Français et 3,0 pour les Anglo-Arabes. Les juments qui ne sortent qu'une année ne représentent que $32 \%$ et $34 \%$ de l'ensemble. L'avantage des juments Selle-Français testées en compétition est de 3,7 points en moyenne : il augmente de 3 à plus de 4 points dans les

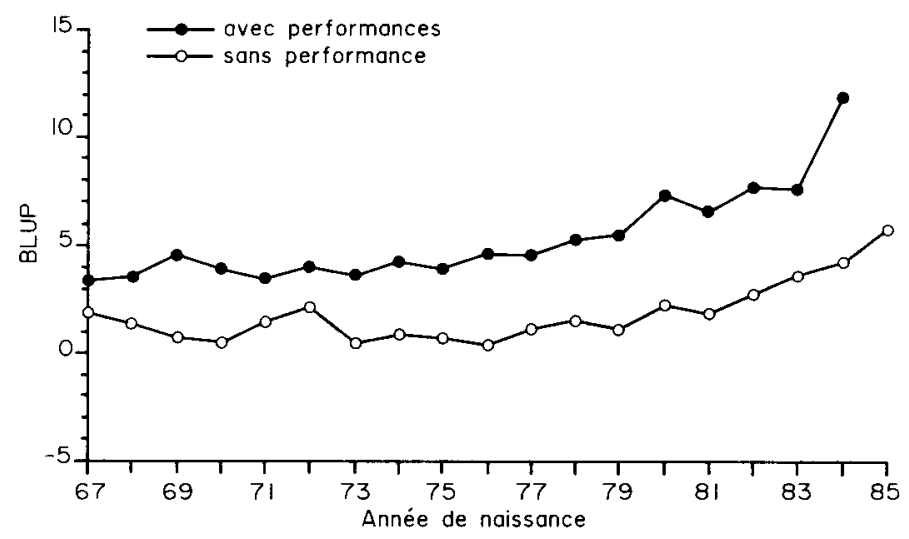

Fig 12. Indice BLUP des poulinières Selle-Français en service en 1988. 


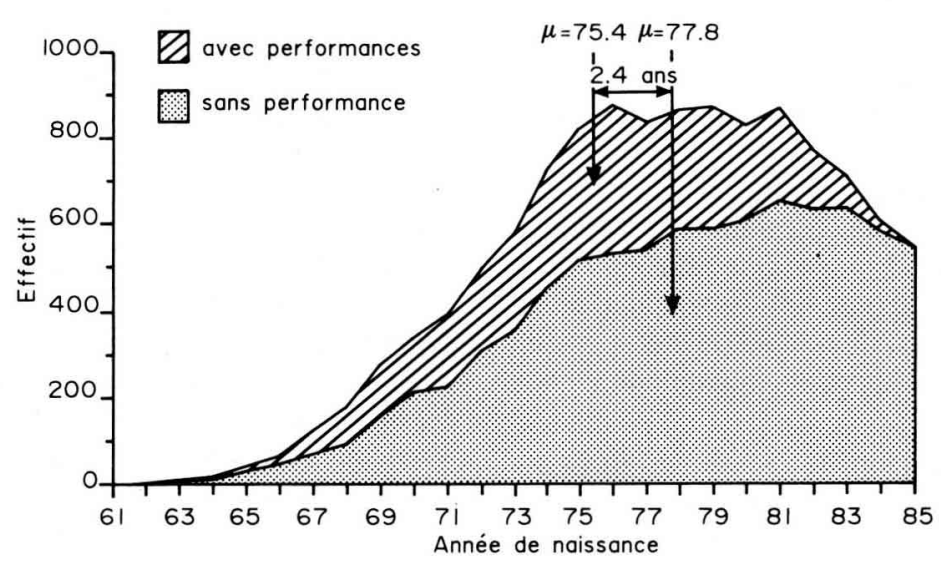

Fig 13. Année de naissance des poulinières Selle-Français en activité en 1988.

dernières années. Chez les Anglo-Arabes, la différence est plus stable et proche de 3 points (fig 12). En revanche ces juments arrivent plus tard à la reproduction que celles qui n'ont pas de performances : contrairement aux étalons, elles ne peuvent mener de front compétition et reproduction. Alors que la présence de performants va de pair chez les mâles avec un rajeunissement de la population, il n'en est pas de même chez les femelles (fig 13). Elles ont en moyenne 2,4 ans de retard (âge moyen 12,3 ans et 10,0 ans pour les Selle-Français et 12,6 ans et 10,2 ans chez les Anglo-Arabes).

\section{DISCUSSION}

La première partie de la discussion portera sur la critique de la réalisation du test de performance. La deuxième partie cherchera à améliorer le plan de sélection des chevaux de concours hippique et proposera des règlès simples de sélection.

\section{Test de performance}

La forte augmentation du nombre d'étalons performants de ces dernières années est principalement due à la mise en place, depuis 1978, d'un circuit de testage à 4 ans des étalons achetés par l'administration à 3 ans. Après ce testage, les chevaux, en majorité, ne sortent plus en compétition : seuls $23 \%$ des étalons Selle-Français et $10 \%$ des Anglo-Arabes ont vraiment réalisé une carrière en compétition d'au moins 3 ans. Les juments suivent, elles, un cursus classique de valorisation en compétition. Cela amène paradoxalement une précision de la sélection sur performance des étalons moindre que celle obtenue pour les poulinières, alors que l'augmentation de cette précision n'entraverait pas leur carrière de reproducteur - contrairement à celle des femelles - s'ils continuaient à être utilisés. Compte tenu de l'héritabilité et de la répétabilité des performances, il est dommage de se priver ainsi de précision sur la voie mâle. La poursuite de la valori-. 
sation en compétition de ces jeunes étalons est le seul moyen d'augmenter cette précision sans nuire à aucun des autres facteurs du progrès génétique. Au contraire, elle peut permettre de diminuer l'intervalle de génération en accélérant la réforme des moins bons performants, non pas forcément grâce à une réforme définitive mais plutôt grâce à une modulation du nombre de descendants que ne manqueront pas d'appliquer les éleveurs au vu des résultats.

L'augmentation de l'intervalle de génération sur la voie femelle due au testage en compétition peut être réduite en limitant ce test à 1 ou 2 ans. Cependant, malgré ce décalage, il faut noter que les juments testées en compétition conservent leur supériorité par rapport à leurs collègues. En effet, leurs 2,4 ans de retard les pénalisent de 0,9 point pour les Selle-Français et de 0,3 point sur les Anglo-Arabes, compte tenu des progrès génétiques réalisés dans chacune de ces races. Elles conservent donc une supériorité de 2,8 et 2,7 points respectivement.

La supériorité des étalons performants correspond au niveau moyen des $54 \%$ meilleures naissances de Selle-Français et des $61 \%$ meilleures naissances d'Anglo-Arabes, alors qu'ils devraient se trouver dans les $2 \%$ meilleures naissances. Chez les femelles, les taux sont respectivement de $78 \%$ et $66 \%$. Si on n'utilisait pour la reproduction que ces juments performantes, on ne serait pas très éloigné du taux de sélection de $60 \%$ nécessaire actuellement au renouvellement de cette population en expansion. Toutefois, cette sélection des femelles ne peut être réellement efficace que si elle est vraiment appliquée à l'ensemble de la population avec la même exigence.

En conclusion, le contrôle des performances en compétition est bien réalisé chez les juments où il pourrait cependant être raccourci (en utilisant prioritairement le "cycle classique" réservé aux jeunes chevaux) et mériterait d'être étendu. Chez les étalons, il est nécessaire d'en augmenter très fortement l'intensité et la précision. On peut aussi regretter que ce contrôle de performance soit à la fois moins fréquent et moins efficace chez l'Anglo-Arabe que chez le Selle-Français. De plus, ce contrôle de performance permet tout à fait l'introduction dans le cheval de concours hippique d'autres races que le SelleFrançais et l'Anglo-Arabe, puisqu'il est le seul moyen d'évaluer la potentialité d'étalons de croisement comme le Pur-Sang ou de juments d'origine peu connue de race "Cheval de Selle" (CS).

\section{Plan de sélection}

La sélection pratiquée actueliement pour produire un cheval de concours hippique peut être nettement améliorée. L'établissement d'un plan de sélection précis nécessite un travail important de simulation des cycles de la population conjointement avec son estimation. C'est uniquement ainsi qu'on peut déterminer de façon optimale le nombre de reproducteurs en service sélectionnés soit sur leurs apparentés, soit sur leurs performances, soit sur leur descendance. Cependant, on peut déjà proposer quelques règles simples capables d'augmenter sensiblement le progrès génétique.

En Selle-Français, comme en AngloArabes, la sélection peut être plus rigoureuse en appliquant une sélection par troncature sur l'indice BLUP avec les mêmes taux de sélection que ceux pratiqués actuellement. De cette façon, la différentielle de sélection qui représente l'écart entre la moyenne des individus sélectionnés et la moyenne de la population est optimale. Ainsi, seuls $1,3 \%$ des Selle-Français et $3,6 \%$ des Anglo-Arabes deviennent éta- 
lons. En appliquant ces taux de sélection à la distribution des indices de chacune des 2 races à la naissance, la supériorité moyenne attendue des étalons est de $+16,3$ et $+8,7$ respectivement. Chez les femelles, le même raisonnement avec un taux de sélection qui avoisine les $60 \%$ $157 \%$ des naissances Selle-Français et $59 \%$ des naissances Anglo-Arabes de 1974 sont devenues poulinières en 1987) permet d'espérer une supériorité moyenne de $+4,1$ points et $+2,6$ points respectivement. Les niveaux minimaux de sélection correspondants sont de $+14,1$ points (SF) et $+7,1$ points (AA) au-dessus de la moyenne des naissances pour les étalons, et de $-1,6$ points (SF) et $-1,0$ point (AA) en dessous de la moyenne des naissances pour les juments. Ensuite, il faut envisager d'appliquer les mêmes règles, obtenues grâce au calcul ci-dessus, aux races extérieures au Selle-Français et à l'Anglo-Arabe, puisqu'elles sont en moyenne d'un niveau inférieur pour le concours hippique. Les progrès génétiques attendus en utilisant les différentielles de sélection précédentes et sans sortir de chaque race sont de 10,2 points par génération en Selle-Français et de 5,7 points en Anglo-Arabes, ce qui correspond à des progrès annuels de 0,85 point et de 0,47 point (avec un intervalle de génération de 12 ans). Ces chiffres multiplient par plus de 2 pour le Selle-Français et plus de 3 pour l'Anglo-Arabe les progrès actuels. Même en supprimant la sélection qui pourrait être pratiquée chez les femelles - qu'il est difficile de contrôler - les progrès génétiques peuvent être doublés.

Pour le Selle-Français, les recommandations pour obtenir les améliorations proposées évoluent chaque année en fonction du progrès génétique. Actuellement, par rapport à la population née en 1987, il faudrait que le BLUP des étalons amenés à produire du Selle-Français soit $\geq+19$ et que celui des poulinières $\geq+4$ (indices arrondis). Pour les étalons, on peut moduler cette exigence en fonction du coefficient de détermination. Cette modification n'optimise pas le progrès génétique mais permet de faire intervenir la notion de risque dans le choix de ces étalons. Ainsi, on peut définir les exigences de cette manière : être sûr à la fois que l'étalon n'est pas détériorateur, et qu'il puisse être dans les $1,3 \%$ meilleurs (ce qui est l'objectif final). L'intervalle de confiance de l'indice est donné par :

$$
\text { Intervalle }=\text { estimation } \pm t \alpha_{2} \sqrt{(1-C D) \sigma_{u}}
$$

avec $\alpha$ : risque que l'intervalle ne contienne pas la vraie valeur du cheval.

La première exigence revient à :

$$
\text { Indice } \geq 5,3+t \alpha_{2} \sqrt{(1-C D) \sigma_{u}}
$$

et la deuxième :

$$
\text { Indice } \geq 19,4-t \alpha_{2} \sqrt{(1-C D) \sigma_{u}}
$$

Une courbe définissant les zones d'indice possibles en fonction du coefficient de détermination est donnée avec un risque de $10 \%$ (fig 14). On constate au début que les exigences sont moins fortes au fur et à mesure que la précision augmente (il faut environ +18 quand on n'a que des apparentés, + 16 quand on a quelques performances) : on est de plus en plus facilement sûr que l'étalon n'est pas détériorateur. En revanche, quand il commence à être bien connu, l'étalon doit être vraiment bon pour atteindre les 1,3\% meilleurs. Parmi les étalons autorisés pour la monte 1988, 97 remplissent les conditions, 3 sur apparentés (CD $\leq 0,36), 52$ avec performances (ou peu de descendants : $0,36<C D \leq 0,55$ ) et 42 avec des descendants $(C D>0,55)$. On peut, de plus, remarquer que d'autres chevaux répondant à ces critères pourraient être sélectionnés et venir aider leurs confrères. Par exemple, pour la seule année de naissance de 1982, 50 mâles sont sélection- 


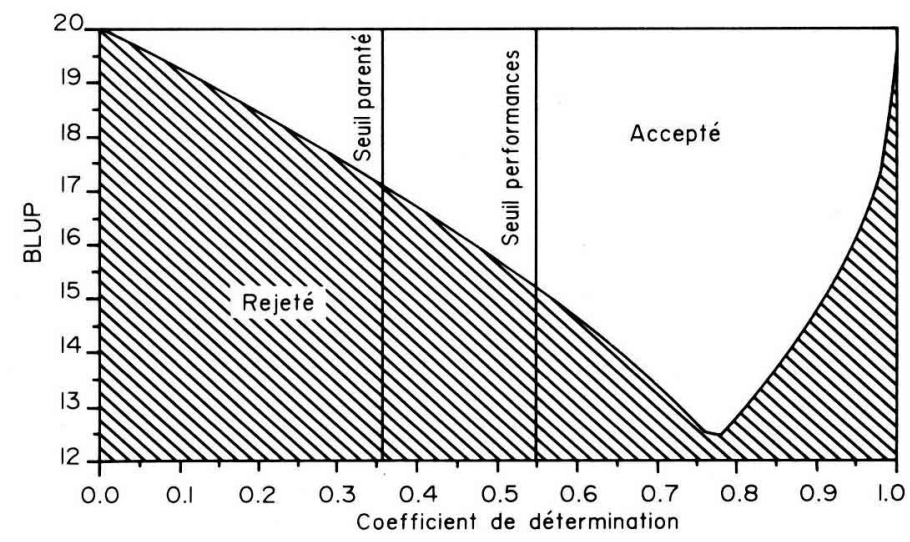

Fig 14. Critère de choix des étalons Selle-Français pour le concours hippique.

nables, dont 9 sur ascendance et 41 sur performances ( 3 années au plus). Un nouveau pas en avant ne pourra ensuite être obtenu qu'avec le changement des structures de reproduction, notamment grâce à l'utilisation de l'insémination artificielle.

L'insémination artificielle permet le développement d'élevages de bon niveau hors de la zone normande, puisque l'une des principales contraintes de l'élevage est la conduite de la jument à la saillie d'étalons stationnés, eux, en poste fixe. Elle permet aussi le raccourcissement de l'intervalle de génération par l'utilisation simultanée de l'étalon en compétition et à la reproduction. Comme nous venons de le voir, ces effets se font déjà sentir. En revanche, la principale influence de l'insémination artificielle sur l'augmentation du niveau moyen de la population grâce à la diffusion des meilleurs étalons est encore quasiment inexistante. La réglementation française limite à 100 saillies les meilleurs étalons, ce qui peut, de toute manière, être obtenu en monte naturelle. De plus, bien peu d'étalons profitent de cette possibilité. En 1988, 11 étalons ayant un BLUP supérieur ou égal à 19 ont fait plus de 60 saillies et seuls 4 d'entre eux avoisinent les 100. Ces mesures, comme la limitation à 25 cartes de saillies en congelé pour les étalons nationaux, interdisent actuellement à l'insémination artificielle de jouer un rôle dans la diffusion du progrès génétique. Pourtant, aujourd'hui même, les 97 étalons en service, qui remplissent les conditions du critère de sélection que nous venons de décrire, auraient suffi à engendrer la génération suivante. Une moyenne de 150 inséminations par étalon ou bien 250 pour les mieux connus $(C D>0,55)$ et 50 pour les jeunes $(C D \leq 0,55)$ le permettrait. Avant même d'intensifier la sélection sur les futurs reproducteurs, on obtenait ainsi, grâce au changement dans la structure de reproduction, une supériorité génétique des étalons de $+13,8$ points, soit à seulement 2,5 points de l'objectif à atteindre.

Pour l'Anglo-Arabe, la situation se présente différemment. Pour continuer à produire un cheval de concours hippique, il présente 3 handicaps supplémentaires. Le premier est son manque de spécialisation qui entraîne une faible détermination pour le concours hippique d'une partie de la population et limite les possiblités de sélection. Le deuxième est que la sélection y est moins intense que dans la race Selle- 
Français puisqu'on y conserve plus de reproducteurs qu'il est nécessaire au renouvellement de la population, du fait de son utilisation en croisement $(3,6 \%$ pour les étalons contre $1,3 \%$ en Selle-Français). Cela diminue, là aussi, l'efficacité de la sélection. Enfin, le troisième handicap est l'exportation des meilleurs reproducteurs hors de la race puisque ceux-ci produisent en Selle-Français. Si l'Anglo-Arabe veut maintenir sa place en concours hippique, alors qu'il présente dès maintenant un retard et qu'il évolue moins vite que le SelleFrançais, il doit pallier ces 3 handicaps. II semble toutefois que le mieux pour cet élevage serait de redéfinir son image en la différenciant nettement de celle du SelleFrançais.

\section{CONCLUSION}

L'indexation généralisée de la population chevaline susceptible de faire du concours hippique est un outil précieux pour l'éleveur qui permet aussi et surtout de mesurer et d'orienter la sélection. L'élevage du cheval de sport est en mutation. Face au développement des sports équestres, s'est créée une nouvelle dynamique : des particuliers souvent extérieurs au monde de l'élevage investissent aujourd'hui dans une jumenterie d'élite ou dans l'étalonnage privé. II est donc d'autant plus important de savoir où l'on va et de mesurer l'importance de la tâche. L'engouement actuel pour le concours hippique crée une demande de plus de 4000 chevaux par an. L'ensemble de la production du SelleFrançais est donc concerné, contrairement à la situation d'il y a 10 ans. Le noyau de sélection issu de la Manche ne peut plus fonctionner seul, et commence d'ores et déjà à voir diminuer son avance génétique sur le reste de la production. Seules les règles rigoureuses de sélection telles qu'elles ont été présentées peuvent per- mettre une accélération du progrès génétique. Cela va de pair avec une augmentation du nombre de saillies par étalon pour augmenter la rapidité de l'évaluation et la diffusion des meilleurs éléments. La valorisation économique doit se faire en recherchant l'augmentation de la qualité du produit et non sa rareté. A l'opposé, l'AngloArabe est en crise. Actuellement, il est en retard pour le concours hippique, face au Selle-Français, et ce retard ne peut que s'accentuer puisqu'il progresse moins vite. II semble urgent et nécessaire de redéfinir ses objectifs. C'est en précisant clairement l'ambition de sélection du Selle-Français vers le saut d'obstacles qu'on offre aux autres la possibilité de se diversifier : il ne faut pas sélectionner tout à la fois, au détriment des progrès de chaque caractère, mais l'on doit au contraire bien personnaliser chaque orientation en en définissant parfaitement les objectifs. C'est dans cette direction déjà largement adoptée déjà par la pratique, que l'élevage français a les meilleurs atouts face à la concurrence sur la marché mondial du cheval de sport. Les orientations de ses concurrents, trop "polyvalentes", lui laissent en effet le champ libre sur le marché le plus important ef le plus rémunérateur qui est celui du cheval de concours hippique. II serait dommage de ne pas valoriser correctement les moyens dont il dispose dans ce domaine.

\section{RÉFÉRENCES}

Bruns $E$ (1987) Estimation of breeding values in riding horses and its practical application. In: 38th Annual Meeting of the EAAP, Lisbon, Portugal, Sept 28-Oct 1st 1987, Study Commission of horse production

Bruns $E$ (1989) Breeding programmes in German riding horses breeding. In: 40th Annual Meeting of the EAAP, Dublin, Ireland, Sept 28-31 1989, Study Commission of horse production 
Hedebro-Velander I, Graaf K, Philipsson J (1989) Breeding programmes for riding horses and thoroughbreds in Sweden. In: 40th Annual Meeting of the EAAP, Dublin, Ireland, Sept 28-31, 1989, Study Commission of horse production

Huizinga HA, Van Der Meij GJW (1988) Paramètres génétiques des performances en saut d'obstacle et en dressage dans la population des chevaux de sport aux Pays-Bas. In: Proc Colloque International "Amélioration Génétique du Cheval de Sport», CEREOPA, Paris, 9 mars 1988

Huizinga HA, Korver $S$, Van Der Meij GJW (1989) Alternatives for the breeding program of riding horses in The Netherlands. In: 40 th Annual Meeting of the EAAP, Dublin, Ireland, Sept 28-31, 1989, Study Commission of horse production

Jensen P, Staun H (1989) Estimation of breeding values of Danish riding horses. In: 40 th Annual Meeting of the EAAP, Dublin, Ireland, Sept 28-31, 1989, Study Commission of horse production

Langlois B (1980) Estimation de la valeur génétique des chevaux de sport d'aprés les sommes gagnées dans les compétitions équestres françaises. Ann Genet Sel Anim $12,15-31$
Meinardus H, Bruns E (1987) BLUP procedure in riding horses based on competition results. In: 38th Annual Meeting of the EAAP, Lisbon, Portugal, Sept 28-Oct 1st 1987, Study Commission of horse production

Philipsson J, Arnason T, Henriksson (1988) Analyse des données concernant les chevaux de selle Suédois et application dans le programme de sélection. In: Proc Colloque International "Amélioration Génétique du Cheval de Sport", CEREOPA, Paris, 9 mars 1988

Tavernier A (1985) BLUP horse story. Rapport de DEA de génétique quantitative et appliquée, université de Paris XI

Tavernier A (1986) Données nouvelles sur les performances des chevaux de sport : précocité, effets maternels, influence du type génétique. In: Journées de la recherche chevaline organisées par le CEREOPA, Paris, 12 mars 1986

Tavernier A (1988) Advantages of BLUP animal model for breeding value estimation of horses. Livest Prod Sci 20, 149-160

Van Raden PM, Freeman AE (1985) Rapid method to obtain bounds on accuracies and prediction error variances in mixed models. J Dairy Sci 68, 2113-2133 